\title{
Enhancement of Vision by Monocular Deprivation in Adult Mice
}

\author{
Glen T. Prusky, ${ }^{1}$ Nazia M. Alam, ${ }^{1}$ and Robert M. Douglas ${ }^{2}$ \\ ${ }^{1}$ Department of Neuroscience, University of Lethbridge, Lethbridge, Alberta, Canada T1K 3M4, and 2Department of Ophthalmology and Visual Sciences, \\ University of British Columbia, Vancouver, British Columbia, Canada V5Z 3N9
}

\begin{abstract}
Plasticity of vision mediated through binocular interactions has been reported in mammals only during a "critical" period in juvenile life, wherein monocular deprivation (MD) causes an enduring loss of visual acuity (amblyopia) selectively through the deprived eye. Here, we report a different form of interocular plasticity of vision in adult mice in which MD leads to an enhancement of the optokinetic response (OKR) selectively through the nondeprived eye. Over $5 \mathrm{~d}$ of MD, the spatial frequency sensitivity of the OKR increased gradually, reaching a plateau of $\sim 36 \%$ above pre-deprivation baseline. Eye opening initiated a gradual decline, but sensitivity was maintained above pre-deprivation baseline for 5-6 d. Enhanced function was restricted to the monocular visual field, notwithstanding the dependence of the plasticity on binocular interactions. Activity in visual cortex ipsilateral to the deprived eye was necessary for the characteristic induction of the enhancement, and activity in visual cortex contralateral to the deprived eye was necessary for its maintenance after MD. The plasticity also displayed distinct learning-like properties: Active testing experience was required to attain maximal enhancement and for enhancement to persist after MD, and the duration of enhanced sensitivity after MD was extended by increasing the length of MD, and by repeating MD. These data show that the adult mouse visual system maintains a form of experience-dependent plasticity in which the visual cortex can modulate the normal function of subcortical visual pathways.
\end{abstract}

Key words: optokinetic response; OKR; visual plasticity; learning and memory; ocular dominance; mouse

\section{Introduction}

A classic finding of mammalian visual plasticity studies is that monocular deprivation (MD) causes lasting physiological effects, a shift of ocular dominance (OD) away from the deprived eye in binocular primary visual cortex (V1) (Wiesel and Hubel, 1963; Hubel and Wiesel, 1970), and behavioral changes, a loss of spatial vision (amblyopia) through the deprived eye (Giffin and Mitchell, 1978), only if deprivation occurs during a "critical" period in juvenile life. Recent physiological studies in mice (Sawtell et al., 2003; Pham et al., 2004; Hofer et al., 2006) have challenged the notion of a restricted period of OD plasticity in cortex by showing that MD in adulthood causes a transient enhancement of responses selectively through the nondeprived eye. A major obstacle to elucidating the function of adult OD plasticity is that no change in visual behavior after adult MD has been reported.

We tested for such changes using a visual perception task previously (Prusky and Douglas, 2003) and found no evidence that adult mouse MD affects visual acuity. However, generating psychometric functions in mice takes longer than the duration of the enhanced physiological responses that have been reported after adult MD. In addition, an enhancement of acuity may not be detectable in a visual perception task in which the threshold is

Received April 6, 2006; revised Sept. 22, 2006; accepted Sept. 22, 2006.

This work was supported by a Discovery Grant from the Natural Sciences and Engineering Research Council of Canada (G.T.P.). We thank Valerie Lapointe, Byron Silver, and Wayne Tschetter for their assistance with this study. Correspondence should be addressed to Glen T. Prusky at the above address. E-mail: prusky@uleth.ca. DOI:10.1523/JNEUROSCI.3396-06.2006

Copyright $\odot 2006$ Society for Neuroscience $\quad$ 0270-6474/06/2611554-08\$15.00/0 thought to be at or near the sampling limit set by retinal anatomy. Furthermore, because the physiological manifestation of OD plasticity in juvenile and adult mice is different, their associated behavioral effects may differ as well.

Recently, we developed a virtual optokinetic system (VOS) for mice (Prusky et al., 2004) and reasoned that because spatial frequency thresholds quantified with this system $[\sim 0.4$ cycles/degree $(\mathrm{c} / \mathrm{d})]$ are normally lower than those generated in mouse perceptual tasks (0.5-0.6 c/d) (Prusky et al., 2000), it could provide the "headroom" to detect an enhancement of function after adult MD. It is often assumed that the optokinetic response (OKR) is controlled only through direct projections from the retina to the accessory optic system (AOS). However, there are indirect pathways to the AOS via the visual cortex, which in other species, mediate binocular responses to stimuli of high spatial frequency and velocity (Grasse et al., 1984; Simpson, 1984; Grasse and Cynader, 1988; Distler and Hoffmann, 1992; Masson et al., 2001; Hoffmann et al., 2002). It is noteworthy that corresponding pathways are present in laboratory rodents (Natal and Britto, 1988; Schmidt et al., 1993; Shintani et al., 1999). We have previously reported that removal of visual cortex in adult mice does not affect OKR sensitivity (Douglas et al., 2005), indicating that these pathways may have little influence on the OKR under normal binocular viewing conditions. We postulate here, however, that the corticofugal projections could become more important as a result of cortical plasticity induced by MD. In addition, because OKR sensitivity can be measured through each eye separately in only a few minutes (Douglas et al., 2005) with the VOS, 
eye-specific responses could be measured before, during, and after MD, allowing us to follow the time course of changes in ways not feasible with current physiological approaches.

\section{Materials and Methods \\ Animals}

C57BL/6 mice of both sexes, bred and reared at the University of Lethbridge from stock originally obtained from the The Jackson Laboratory (Bar Harbor, ME), were used as subjects. Animals ranged in age from $60-300 \mathrm{~d}$ at the time of testing. Experimental procedures were approved by the University of Lethbridge Animal Care Committee and conducted in accordance with the standards of the Canadian Council on Animal Care.

\section{Virtual optokinetic system}

A virtual cylinder covered with a vertical sine wave grating was projected in three-dimensional coordinate space on a quadrangle of computer monitors around a testing arena (Prusky et al., 2004). Individual mice were placed on a round, elevated platform in the center of the arena where they were allowed to move freely. The position of the animal's head was tracked continuously with the aid of a computer mouse and a crosshair superimposed on a video image of the arena. The $x-y$ positional coordinates of the crosshair in the video frame dictated the hub of the virtual cylinder, thereby enabling the cylinder wall to be maintained at a constant "distance" from the animal's viewing position and effectively "clamping" the spatial frequency of the stimulus. When the cylinder was rotated $(12 \%)$ and the mouse followed with corresponding horizontal head and neck movements (OKR), it was judged that the animal's visual system could distinguish the grating.

\section{Determination of visual thresholds}

When measuring the spatial frequency threshold of the OKR, a homogeneous gray stimulus was projected on the cylinder at the beginning of each testing session. The experimenter waited until the animal stopped moving, at which time the gray was switched to a low spatial frequency $(\sim 0.04 \mathrm{c} / \mathrm{d})$, high-contrast $(\sim 100 \%)$ sine wave grating moving in one direction. The animal was assessed for tracking behavior for a few seconds, after which the gray stimulus was restored. This procedure was repeated until unambiguous examples of tracking were observed. The short testing epochs reduced the possibility of the mouse adapting to the stimulus (it established that each animal was capable of tracking when a salient stimulus was present), and initiating the testing at a low spatial frequency enabled each animal's OKR to be typified. The spatial frequency of the grating was then increased incrementally until the animal no longer tracked. The process was repeated at least three times until the highest spatial frequency that the mouse could track was established unequivocally. Occasional squeaking noises or finger tapping on the lid of the apparatus were used to induce the animal to change its position or stop moving, which facilitated more rapid testing.

Thresholds through each eye were measured separately by reversing the rotation of the cylinder. When one eye is temporarily occluded, only motion in the temporal-to-nasal direction for the other eye evokes tracking; when the maximal spatial frequency capable of driving a response is measured separately under monocular and binocular viewing conditions, the monocular threshold is identical to the binocular threshold measured with the same direction of rotation (Douglas et al., 2005).

A contrast sensitivity function was established using the general procedures described above, except that the contrast at a preselected spatial frequency was systematically reduced until the minimum contrast to evoke tracking was identified. A contrast threshold was measured at six spatial frequencies: 0.031, 0.064, 0.092, 0.103, 0.192, 0.272 (c/d). Contrast sensitivity was calculated at each spatial frequency as a Michelson contrast from the screen luminances $(\max -\min ) /(\max +\min )$ and the reciprocal of the threshold (mean white, $253.273 \mathrm{~cd} / \mathrm{m}^{2}$; mean black, $0.255 \mathrm{~cd} / \mathrm{m}^{2}$ ).

Whenever possible, experimenters were blind to the animal's treatment condition or previously recorded thresholds, and thresholds were regularly validated independently by more than one observer. Mice were normally tested during the first $6 \mathrm{~h}$ of their daylight cycle $(12 \mathrm{~h}$ light/dark cycle; light on at 7:30 A.M.), and a complete set of spatial frequency and contrast thresholds for an animal through both eyes were generated in $10-20 \mathrm{~min}$.

Experimental groups were tested once per day unless otherwise noted. There was very little variability, and repeated-measures ANOVAs (SPSS; Kaleidagraph) on the time series, followed by Tukey's honestly significant difference tests using a $p<0.05$ criterion were used to decide whether differences across days were significant.

\section{Binocular and monocular visual field thresholds}

Although mice lack a specialized fovea, they regularly "foveate" (i.e., moving their head to place information of interest in their binocular field), indicating that binocular and monocular visual functions differ. To quantify behavioral correlates of this bias, we measured spatial frequency thresholds within restricted segments of the visual field. The general procedures described above were used, with the addition that the drifting grating was limited to only part of the visual field. Although mice are unrestrained during testing, their OKRs are effectively limited to a plane parallel to the horizon. We took advantage of this constraint and monitored gaze by continuously tracking the horizontal orientation of the head with the aid of a line superimposed on the video frame and oriented along the snout. The vector generated by this procedure was used to maintain in real time the grating patch at a constant angle relative to the head. The central $30-40^{\circ}$ of the upper portion of each visual hemifield $\left(\sim 110^{\circ}\right.$ from midline) in mice has the potential to receive input from both eyes (Wagor et al., 1980). Because cells in V1 with receptive fields outside the central $25^{\circ}$ in each hemifield are less likely to be driven by the ipsilateral eye (Gordon and Stryker, 1996), for binocular field testing we conservatively defined the binocular zone as, and limited our stimulation to, $50^{\circ}$ straddling the midline. We also measured thresholds within the monocular zone (partial: $50^{\circ}$ centered at $65^{\circ}$ from the midline; full: $75^{\circ}$ centered at $37.5^{\circ}$ from the midline) and across both fields ( -25 to $110^{\circ}$ from the midline).

\section{Visual deprivation}

Under general anesthetic (inhalated isoflurane; induction at 2.5-4.5\%, maintenance at $1-2 \%$ evaporated in $1-1.5 \mathrm{~L} / \mathrm{min}_{2}$ ), a topical antibacterial ophthalmic agent [bacitracin-neomycin-polymyxin (BNP)] was applied to the eyes, the middle $3.0-4.0 \mathrm{~mm}$ of an eyelid was trimmed and sewn $(6.0$ silk) shut with one or two mattress stitches placed below the eyelid margin, and the knot was sealed with cyanoacrylate glue. After surgery, a topical anesthetic ( $0.5 \%$ proparacine hydrochloride) was applied to the sutured eye and the animal was recovered on a warm blanket. At the end of a preselected deprivation period, animals were reanesthetized and the stitches were removed. Eyes were checked regularly for clarity, and animals with optical abnormalities were removed from the study.

\section{Enucleation}

The same general procedures as above were used, except that eyelids were not manipulated and an eye was removed. The connective tissue around the sclera was carefully removed to expose the optic nerve. The nerve was then cut, the eye was extracted, and the orbit was packed with sterile gelfoam. Animals were recovered as described above.

\section{Visual cortex surgery}

Bilateral visual cortex aspirations. Mice were anesthetized as above. After placement in a stereotaxic frame, ophthalmic ointment (BNP) was applied to the eyes and the top of the head was washed with sterile saline and wiped with dilute Hibitane and $70 \%$ ethanol. A midline incision was made in the scalp, the skin was resected to expose the skull, and the boundaries of striate cortex were demarcated on the skull with reference to stereotaxic coordinates (Franklin and Paxinos, 1997). A dental drill was used to create a craniotomy ( $1.5 \mathrm{~mm}$ lateral to $4.0 \mathrm{~mm}$ lateral from midline; 2.5 to $-5.0 \mathrm{~mm}$ posterior from bregma) in both hemispheres, the dura was resected, and cortex within the trephinations was aspirated down to white matter. The incision in the scalp was closed with stitches, animals were injected with analgesic (Metacam; 0.04 cc, i.p.; $0.25 \mathrm{mg} / \mathrm{ml}$ ) and recovered on a warm blanket, and were returned to their home cage once they were mobile and alert. 
Table 1. Summary of major results with descriptive and inferential statistics

\begin{tabular}{|c|c|c|c|c|c|c|c|c|}
\hline Group & $N$ & Baseline & Maximum & $\%$ & Days & $F$ & $\mathrm{df}$ & Figures \\
\hline \multicolumn{9}{|l|}{ No change } \\
\hline $5 \mathrm{~d}$ binocular deprivation & 5 & $0.388 \pm 0.0009$ & $0.386 \pm 0.0018$ & 0 & 0 & 1.14 & 4,6 & $3 D$ \\
\hline $5 \mathrm{~d} M D+$ ipsilateral muscimol & 5 & $0.392 \pm 0.0004$ & $0.390 \pm 0.0012$ & 0 & 0 & 1 & 5,31 & $4 B$ \\
\hline \multicolumn{9}{|l|}{ Small enhancement $(\sim 10 \%)$} \\
\hline Enucleation & 4 & $0.393 \pm 0.0005$ & $0.434 \pm 0.0017$ & 10 & NA & 115 & 3,15 & $3 E$ \\
\hline $5 \mathrm{~d} M \mathrm{MD}+$ bilateral lesions & 6 & $0.388 \pm 0.0021$ & $0.430 \pm 0.0009$ & 11 & 0 & 130 & 5,14 & $4 A$ \\
\hline \multicolumn{9}{|c|}{ Intermediate enhancement ( $\sim 28 \%)$; no persistence } \\
\hline $5 \mathrm{~d} M D+$ without testing & 6 & $0.387 \pm 0.0021$ & $0.497 \pm 0.0000$ & 29 & 0 & 2017 & 4,7 & $3 A$ \\
\hline $10 \mathrm{~d} \mathrm{MD}+$ without testing & 6 & $0.388 \pm 0.0015$ & $0.495 \pm 0.0006$ & 28 & 0 & 4469 & 5,7 & $3 B$ \\
\hline \multicolumn{9}{|l|}{ Maximal enhancement ( $\sim 37 \%)$; no persistence } \\
\hline $3 \mathrm{~d} M D$ (first) & 5 & $0.389 \pm 0.0009$ & $0.530 \pm 0.0007$ & 36 & 0 & 14769 & 5,10 & $5 A, 5 D$ \\
\hline $3 \mathrm{~d} \mathrm{MD}$ (second) & 5 & $0.388 \pm 0.0009$ & $0.527 \pm 0.0015$ & 36 & 0 & 4841 & 4,10 & $6 A, 6 D$ \\
\hline $5 \mathrm{~d}$ MD + contralateral muscimol & 6 & $0.385 \pm 0.0006$ & $0.531 \pm 0.0018$ & 37 & 0 & 2185 & 5,31 & $4 C$ \\
\hline \multicolumn{9}{|c|}{ Maximal enhancement ( $\sim 37 \%)$; with short persistence } \\
\hline $5 \mathrm{~d} M D$ (first) & 5 & $0.395 \pm 0.0014$ & $0.5372 \pm 0.0007$ & 36 & 5 & 1018 & 4,19 & 1 \\
\hline $5 \mathrm{~d} \mathrm{MD}$ (first) & 7 & $0.396 \pm 0.0012$ & $0.5463 \pm 0.0021$ & 38 & 6 & 607 & 6,21 & $1,5 B, 5 D$ \\
\hline $5 \mathrm{~d}$ MD (ipsilateral Elvax control) & 3 & $0.387 \pm 0.0030$ & $0.5310 \pm 0.0015$ & 37 & 6 & 968 & 2,15 & $4 B$ \\
\hline $5 \mathrm{~d}$ MD (contralateral Elvax control) & 2 & $0.387 \pm 0.0023$ & $0.5268 \pm 0.0011$ & 36 & 6 & 6907 & 1,15 & $4 C$ \\
\hline $5 \mathrm{~d}$ MD (after ipsilateral muscimol) & 5 & $0.390 \pm 0.0006$ & $0.5128 \pm 0.0016$ & 31 & 7 & 1117 & 4,16 & $4 B$ \\
\hline $5 \mathrm{~d} \mathrm{MD} \mathrm{(after} \mathrm{contralateral} \mathrm{muscimol)}$ & 6 & $0.392 \pm 0.0005$ & $0.5345 \pm 0.0015$ & 36 & 7 & 1215 & 5,16 & $4 C$ \\
\hline \multicolumn{9}{|c|}{ Maximal enhancement ( $\sim 37 \%)$; with extended persistence } \\
\hline $5 d M D+\times 2$ testing & 5 & $0.389 \pm 0.0013$ & $0.535 \pm 0.0012$ & 37 & 8 & 1589 & 4,25 & $3 C, 5 D$ \\
\hline $10 \mathrm{~d}$ MD (first) & 5 & $0.385 \pm 0.0037$ & $0.530 \pm 0.0016$ & 38 & 11 & 1764 & 4,30 & $5 C, 5 D, 6 E, 6 F$ \\
\hline $5 \mathrm{~d} \mathrm{MD}$ (second) & 7 & $0.396 \pm 0.0021$ & $0.547 \pm 0.0021$ & 38 & 31 & 1541 & 6,30 & $6 B, 6 D, 6 F$ \\
\hline $10 \mathrm{~d} \mathrm{MD}$ (second) & 5 & $0.389 \pm 0.0009$ & $0.538 \pm 0.0007$ & 38 & 36 & 6639 & 4,35 & $6 C, 6 D, 6 E$ \\
\hline $5 \mathrm{~d}$ MD (second after 6 months) & 5 & $0.397 \pm 0.0016$ & $0.548 \pm 0.0026$ & 38 & 66 & 1402 & 4,31 & NA \\
\hline $10 \mathrm{~d} M D$ (third) & 3 & $0.389 \pm 0.0024$ & $0.532 \pm 0.0007$ & 37 & 85 & 346 & 2,39 & $6 E$ \\
\hline
\end{tabular}

Groups are listed (left column) and arranged (subheadings) according to experimental effects. The number of animals in a group ( $N$ ), the baseline spatial frequency sensitivity with SEM (Baseline), the maximum sensitivity over the course of the experiment with SEM (Maximum), the percentage change between baseline and maximum sensitivity (\%), the number of days enhanced sensitivity persists above baseline after MD (Days), Fvalues of repeated-measures ANOVAs for the group $(F)$, degrees of freedom of the analysis (df), and references to figure number and panel of the experiment (Figures) are listed for each group in columns.

Lesion location and size was verified once behavioral testing was completed. Animals were anesthetized and perfused with cold PBS followed by buffered $4 \%$ paraformaldehyde. Once the brains were extracted, they were postfixed in $4 \%$ paraformaldehyde and stored in $30 \%$ sucrose. The dorsal surface of each brain was digitized and fiducial features were drawn, and the area of each lesion was circumscribed and shaded with light gray. The illustrations of each animal's brain were then superimposed on one another.

Unilateral reversible inactivation of visual cortex. Cellular activity in visual cortex was inactivated independently in each hemisphere using muscimol-releasing Elvax. Elvax was prepared using a modification of procedures described previously (Smith et al., 1995, 2004; Prusky and Ramoa, 1999). In brief, beads of the ethylene-vinyl acetate copolymer Elvax 40-W (a gift from DuPont, Wilmington, DE) were washed for $24 \mathrm{~h}$ each in five changes of $95 \%$ alcohol, followed by five changes in $100 \%$ alcohol. Washed beads were decanted, dried overnight at $40^{\circ} \mathrm{C}$, and stored at room temperature. A total of $100 \mathrm{mg}$ of the dried beads was placed in a scintillation tube containing $1 \mathrm{ml}$ of methylene chloride. Once the beads were dissolved, the solution was mixed on a vortex and $20 \mu \mathrm{l}$ of either double-distilled water (for control implants) or an aqueous solution of muscimol was added to the dilute Elvax. Muscimol was dissolved in one equivalent of $10 \mathrm{M} \mathrm{NaOH}_{(\mathrm{aq})}$, and the volume was made up to 20 $\mu l$ with double-distilled water. A volume of $10 \mu \mathrm{l}$ of $5 \%$ fast green $_{(\mathrm{aq})}$ was also added to the mixture, and the entire solution was vortexed for at least $10 \mathrm{~min}$. The uncapped tube was placed upright on dry ice until the solution was frozen, and then transferred to $-80^{\circ} \mathrm{C}$ for $6 \mathrm{~d}$ followed by another $4 \mathrm{~d}$ at $-20^{\circ} \mathrm{C}$. The resulting cylinders were cut into $200 \mu \mathrm{m}$ circular sheets on a freezing microtome and stored on glass slides at $4^{\circ} \mathrm{C}$. Sheets of prepared Elvax were cut into $4 \mathrm{~mm}^{2}$ squares and washed in sterile PBS for at least $1 \mathrm{~h}$ before surgery.

Mice were prepared for surgery as above, with the exceptions that a trephination was made in only one hemisphere, and after removing the dura, an Elvax square was fixed in place on the surface of the cortex by tucking it under the edges of the bone. The effect of reversing cortical inactivation was investigated in some animals by removing the Elvax. In this case, the trephination was exposed as above, and the Elvax was slid out from under the edges of the bone. Incisions in the scalp were closed as above and postsurgical care was identical. On completion of behavioral testing, brains were extracted, prepared as above, and the placement of Elvax was confirmed.

\section{Results}

The manipulations produced large and statistically highly significant changes in the sensitivity of the OKR. Table 1 provides a summary of the statistical analysis, including the number of animals in each group, baseline spatial frequency sensitivity, maximum sensitivity, percentage change in sensitivity, duration of enhancement after an MD in days, $F$ values of repeated-measures ANOVAs for the group with degrees of freedom, as well as references to figures.

\section{Effect of 5 d MD}

We first characterized the effects of $5 \mathrm{~d}$ of MD on spatial frequency and contrast sensitivity thresholds of the OKR through nondeprived and deprived eyes. Spatial frequency sensitivity was stable through both eyes before MD, and no change was observed through the nondeprived eye when it was first tested within $2-3 \mathrm{~h}$ after MD (Fig. 1A). On each of the subsequent $3 \mathrm{~d}$, however, sensitivity increased substantially, and then leveled off at $\sim 36 \%$ above baseline. When the deprived eye was opened, the threshold commenced a gradual decline, but remained above predeprivation baseline for 5-6 $\mathrm{d}$. Thresholds through the deprived eye were slightly depressed in some animals when first measured after the MD, but by the following day, they did not differ from predeprivation baseline. We believe that minor trauma to the eyelids of these animals, which caused them to partially close their eyelids for a 

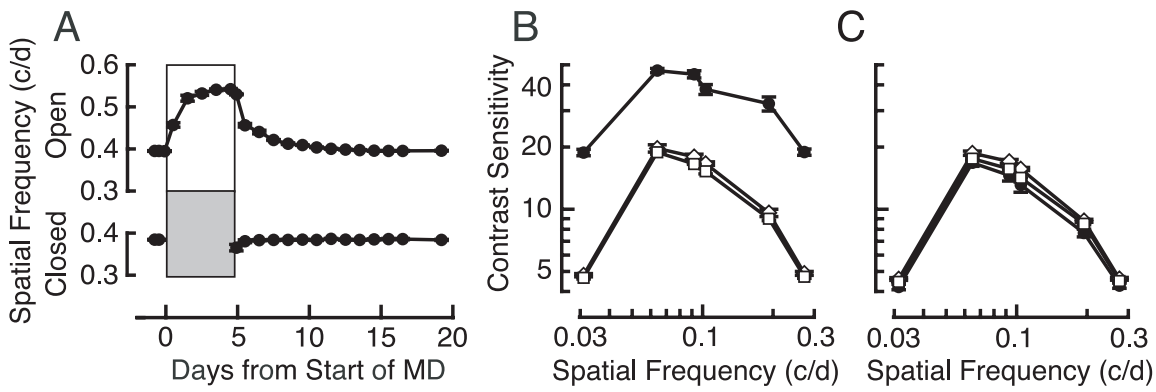

Figure 1. Effect of $5 \mathrm{~d} M D$ (shading) on OKR sensitivity. $\boldsymbol{A}$, Spatial frequency sensitivity through nondeprived eyes (open; top trace) was increased and reached a maximum over 3-4 d of MD. Opening the deprived eye initiated a gradual decline to baseline over 5- $6 \mathrm{~d}$. Deprived eye responses (closed; bottom trace) were not affected after the first day. $\boldsymbol{B}$, Effect of MD on contrast sensitivity was similar: Sensitivity increased from baseline (open squares) over $5 \mathrm{~d}$ of MD (day 5 plotted with closed circles) and returned to baseline levels over $10 \mathrm{~d}$ (open diamonds; plotted is the 10th day after MD). $C$, There was little effect of MD on contrast sensitivity through the deprived eye (pre-deprivation baseline, open squares; immediately after day 5 of MD, closed circles; $10 \mathrm{~d}$ after MD, open diamonds). \pm SEM plotted with vertical scale lines are present but are often occluded by data symbols in this and other figures.
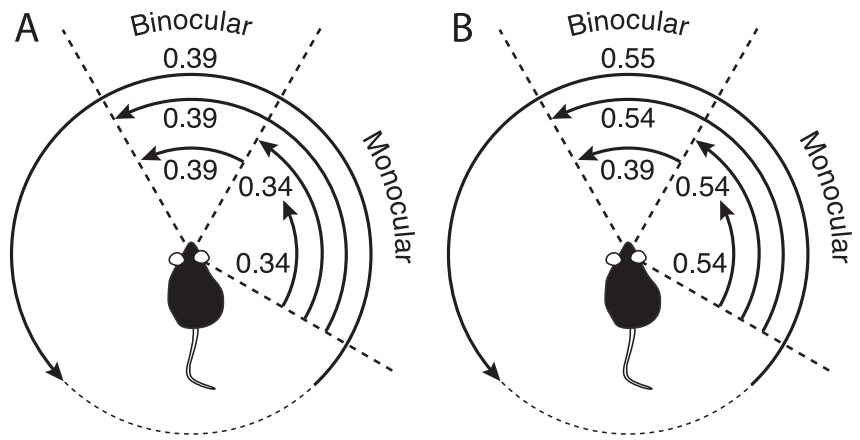

Figure 2. Selective visual field responses in schematic illustration. The length of arching arrows represent the segment of the visual field in which the grating was present during testing (binocular field, Binocular; monocular field, Monocular), and the associated numbers represent spatial frequency thresholds in c/d (see Materials and Methods for details). $A$, In experimentally naive mice, full-field (outside arrow; dotted segment represents the region of the field not visible to the eyes) stimulation generated a threshold of $0.39 \mathrm{c} / \mathrm{d}$. Limiting the stimulus to the binocular field resulted in the same threshold as full-field stimulation $(0.39 \mathrm{c} / \mathrm{d})$; monocularfield responses $(0.34 \mathrm{c} / \mathrm{d})$ were lower regardless of the size of the stimulus within the field. Combining monocular and binocular stimulation resulted in the same threshold $(0.39 \mathrm{c} / \mathrm{d})$ as binocular alone or full-field stimulation. $\boldsymbol{B}$, Full-field stimulation in enhanced mice resulted in a threshold of $0.55 \mathrm{c} / \mathrm{d}$. Monocular field stimulation alone or combined with binocular resulted in about the same threshold $(0.54 \mathrm{c} / \mathrm{d})$, whereas binocular responses alone did not differ $(0.39 \mathrm{c} / \mathrm{d})$ from those in naive animals $(\boldsymbol{A})$.

short period after removal of the suture, is likely responsible for this temporary decrease.

Contrast sensitivity was enhanced with a profile that paralleled that of enhanced spatial frequency sensitivity: There was a general increase in contrast sensitivity at all spatial frequencies during the $\mathrm{MD}$, which then returned to baseline several days after eye opening (Fig. $1 B$ ). Deprivation did not affect contrast sensitivity through the deprived eye (Fig. $1 C$ ).

Changes in contrast sensitivity generally matched changes in spatial frequency sensitivity in other experiments in this study (data not shown). In addition, absolute monocular directionality of the OKR (i.e., only temporal-nasal movement of the stimulus drove OKRs through an eye) (Douglas et al., 2005) was maintained under all experimental conditions.

\section{Responses within binocular and monocular visual fields}

To establish in normal mice whether there is a visual field specialization in the OKR, as well as to determine whether enhanced sensitivity after MD is limited to a specific visual field, we measured spatial frequency thresholds within selected regions of the visual field in experimentally naive $(N=$ $6)$ and enhanced mice $(N=6)$. In normal mice (Fig. $2 A$ ), stimulating the binocular field alone generated the same threshold as full-field stimulation $(0.389 \pm 0.060$ c/d); however, monocular-field responses $\left(50^{\circ}=0.338 \pm 0.052 \mathrm{c} / \mathrm{d} ; 75^{\circ}=0.338 \pm\right.$ $0.052 \mathrm{c} / \mathrm{d}$ ) were lower. When the stimulus was present in both binocular and monocular fields, the threshold $(0.389 \pm 0.060$ c/d) matched that generated with binocular, or full-field stimulation. The same measurements in enhanced mice (Fig. $2 B$, measured at maximal enhancement) revealed that sensitivity within the binocular field remained normal $(0.389 \pm 0.060$ $\mathrm{c} / \mathrm{d})$, but that increased sensitivity in the monocular field accounted for the enhancement; monocularfield testing alone $(0.542 \pm 0.084 \mathrm{c} / \mathrm{d})$ or combined with binocular stimulation resulted in similarly improved responses $(0.544 \pm 0.084 \mathrm{c} / \mathrm{d})$ as full-field testing $(0.545 \pm 0.084 \mathrm{c} / \mathrm{d})$.

\section{Experiential control of enhancement}

We investigated whether enhancement was dependent on the experience of being tested, by not testing the nondeprived eye during a $5 \mathrm{~d} \mathrm{MD}$ until immediately (no more than $30 \mathrm{~min}$ ) before the deprived eye was opened (Fig. 3A). We found that spatial frequency sensitivity did not reach maximal improvement in these animals ( $\sim 29$ vs $36 \%$ in tested animals) (Table 1 ), and that as soon as the deprived eye was opened, sensitivity fell to predeprivation levels. Extending the period of deprivation to $10 \mathrm{~d}$ without testing resulted in a similar profile (Fig. $3 B$ ); sensitivity did not reach maximal improvement and enhanced responses did not persist after MD. Twice daily testing during a $5 \mathrm{~d} \mathrm{MD} \mathrm{(Fig.}$ $3 C$ ) slightly attenuated enhancement during the MD, and moderately increased the magnitude and duration of enhanced responses after MD.

To determine whether MD-induced enhancement of the OKR was the result of a competitive interaction between the eyes, we investigated the effects of $5 \mathrm{~d}$ of binocular deprivation (BD) on OKR sensitivity. BD had little effect on sensitivity (Fig. 3D). To determine whether characteristic enhancement was dependent on the presence of both eyes, or whether a reduction in sensitivity could be induced by deprivation in the absence of a competing eye, we also monocularly enucleated an eye and deprived the intact eye. Monocular enucleation resulted in only a small enhancement after $1 \mathrm{~d}$ (Fig. 3E) [whether intact-eye responses were tested $(10.4 \pm 1.03 \% ; N=4)$ or not (data not shown)], which did not change thereafter. Depriving the intact eye also did not affect sensitivity.

\section{Effects of removing or inactivating visual cortex}

We investigated the necessity of visual cortex in the MD-induced enhancement of OKR sensitivity by removing V1 bilaterally and commencing a $5 \mathrm{~d} \mathrm{MD}$ (Fig. $4 A$ ). Over the first $2 \mathrm{~d}$ of $\mathrm{MD}$, sensitivity increased slightly $(\sim 10 \%)$ and plateaued. When the deprived eye was opened, sensitivity fell immediately to predeprivation baseline. Deprived-eye responses were not affected by the treatment, confirming our previously published results (Douglas et al., 2005) that visual cortex lesions do not 
reduce the spatial frequency sensitivity of the OKR in animals with balanced visual experience.

Having confirmed a role for visual cortex in the MD-induced plasticity of the OKR with V1 ablations, we investigated the specific role of activity in $\mathrm{V} 1$ on the enhancement of OKR sensitivity by inactivating visual cortex before a $5 \mathrm{~d} \mathrm{MD} \mathrm{(Fig.}$ $4 B, C)$. Sensitivity was stable through both eyes before Elvax surgery, and no change was recorded over the course of postsurgical recovery. Within a few hours of surgery, all muscimol-treated animals developed a slight twisting of their head in the direction of the hemisphere with the Elvax, which dissipated within a day. None of the control Elvax animals showed this response. The enhancement that characteristically follows MD was absent in animals with muscimol-releasing Elvax located ipsilateral to the MD (Fig. 4B). Plasticity could be demonstrated subsequently after removal of the Elvax (i.e., a characteristic profile of MD-induced enhancement was generated), indicating that release of muscimol was the causal factor for the initial blockade of enhancement. Note also the absence of the rapid jump to maximal levels usually seen after a second $5 \mathrm{~d}$ MD (see below for details). This was confirmed by placing control Elvax contralateral to the $\mathrm{MD}$, which resulted in an enhancement profile characteristic of that recorded in intact animals.

Muscimol-releasing Elvax placed contralateral to the MD (Fig. 4C) resulted in a profile of enhancement typical of that in animals with control Elvax; however, enhanced responses did not persist after the deprived eye was opened. A characteristic profile of enhancement was also recovered after removal of the muscimol-releasing Elvax.

An examination of the brains after behavioral testing revealed that a small indentation and flattening of the cortex was present in the region where the Elvax was placed, but the tissue appeared otherwise normal.

\section{Effect of varying the duration of MD}

We investigated the relationship between the duration of $\mathrm{MD}$, and the persistence of enhancement after MD by examining the effects on spatial frequency thresholds of periods of MD shorter (3 d) (Fig. 5A), and longer (10 d) (Fig. 5C), than 5 d (Fig. 5B). Three days and $10 \mathrm{~d}$ of MD resulted in a profile of enhancement through the nondeprived eye similar to that during a $5 \mathrm{~d} \mathrm{MD}$ (i.e., increasing sensitivity to a maximum over $2-3 \mathrm{~d}$ ). The profile after $\mathrm{MD}$, however, differed dramatically: There was no persistence of enhanced responses after a $3 \mathrm{~d}$ MD (i.e., immediately on eye opening, sensitivity fell to pre-deprivation levels) (Fig. 5D), and $10 \mathrm{~d}$ of MD resulted in enhanced sensitivity that persisted at maximal levels longer than after a $5 \mathrm{~d} \mathrm{MD} \mathrm{(Fig.} \mathrm{5D).} \mathrm{There} \mathrm{was} \mathrm{no}$ change in sensitivity measured through deprived eyes $1 \mathrm{~d}$ after MD (Fig. $5 A-C$, bottom traces).

\section{Effect of repeated MD}

We investigated whether the MD-induced enhancement of the OKR could be potentiated by repeating a period(s) of MD. Repeating a $3 \mathrm{~d} \mathrm{MD}, 7 \mathrm{~d}$ after the first, resulted in a profile of enhanced sensitivity (Fig. 6A) similar to that after the first $3 \mathrm{~d}$ of MD (Fig. 5A). Repeating a $5 \mathrm{~d}$ period of MD, however, dramatically changed the profile of enhancement: sensitivity jumped to maximal values immediately after the surgery [Fig. $6 B, D$ (data from animals with a 1 month delay between the end of the first $\mathrm{MD}$ and the beginning of the second MD is plotted, but intervals up to 6 months result in a similar profile (Table 1)]. In addition, elevated sensitivity persisted far longer after the second $\mathrm{MD}(>4$ weeks) (Fig. $6 B, E)$ than after the first $(\sim 1$ week) (Fig. $5 B, D)$. A second $10 \mathrm{~d}$ MD resulted in a similar profile of enhancement to that after a second $5 \mathrm{~d} \mathrm{MD}$, but enhanced function was maintained above baseline for over 6 weeks (Fig. 6C,E). A third $10 \mathrm{~d}$ period MD resulted in enhanced sensitivity that endured $>10$ weeks (Fig. $6 E$ ). The duration of enhancement after a single $10 \mathrm{~d}$ MD was also much shorter than after the second of two, $5 \mathrm{~d}$ periods of MD (Fig. $6 F$ ). No change in function through deprived eyes was recorded (Fig. $6 A-C$, bottom traces).

\section{Discussion}

Spatial frequency and contrast sensitivity thresholds of the adult mouse OKR do not normally depend on visual cortex and appear to be limited by balanced interocular interactions. The bias introduced by MD enables an enhancement of sensitivity in the monocular field of the nondeprived eye, which depends on the experience of testing sensitivity of the OKR, and activity in visual 

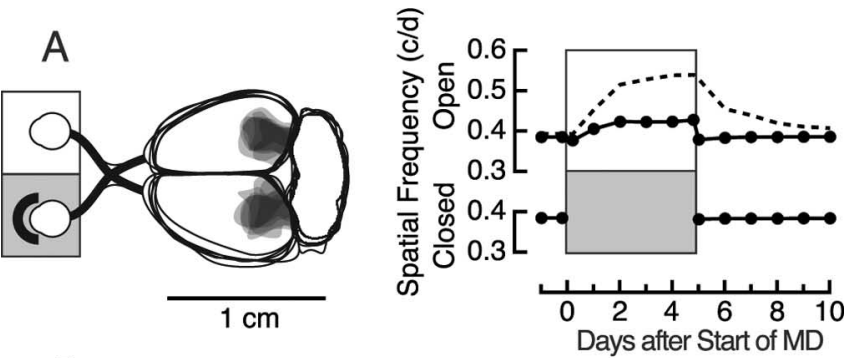

B
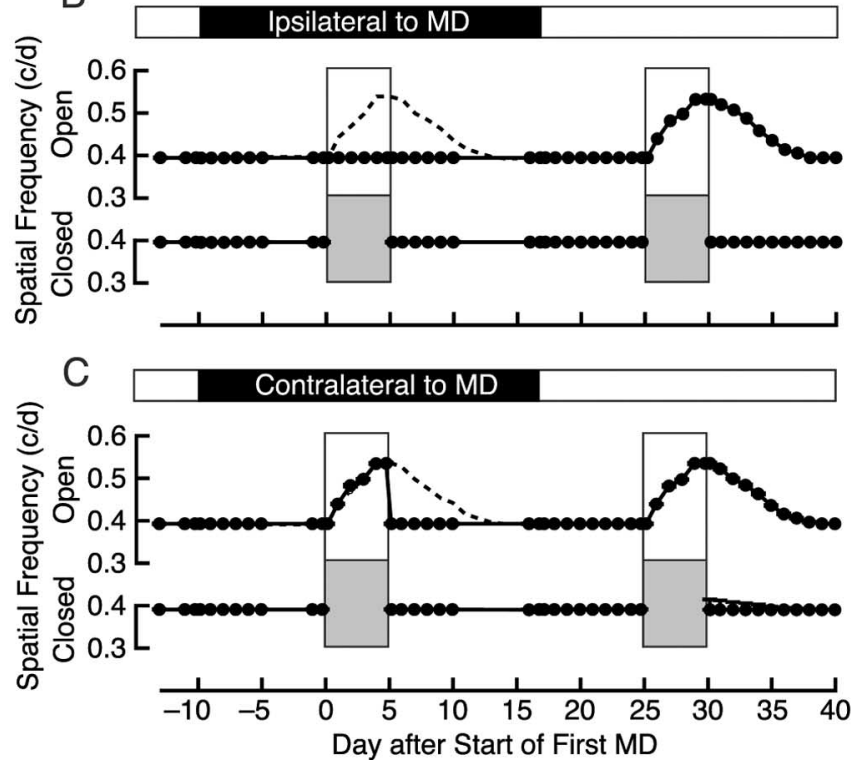

Figure 4. Effect of visual cortex manipulations on enhancement induced by $5 \mathrm{~d}$ MD. A, Bilateral aspiration of visual cortex. Left panel, Schematic representation of deprived (shaded box) and nondeprived eyes combined with superimposed drawings of the brains showing the location of lesions. Right panel, For comparison, the dashed trace is nondeprived eye profile from Figure $1 \mathrm{~A}$. The spatial frequency threshold through the nondeprived eye of animals with bilateral V1 lesions (top trace) became slightly elevated $(\sim 10 \%)$ during the MD (gray box) and dropped to baseline immediately on eye opening. Sensitivity through the deprived eye (bottom trace) was not affected by the treatment. $B, C$, Unilateral inactivation of visual cortex. Period during that muscimol-releasing Elvax was present is indicated in black; period of MD is indicated in gray. Sensitivity measured before surgery was stable at normal baseline levels, and muscimol-releasing Elvax did not affect sensitivity through either eye before MD. $\boldsymbol{B}$, Inactivating visual cortex ipsilateral to the deprived eye blocked the characteristic enhancement of sensitivity (closed circles). The effect of silencing was reversible, however, because MD after removal of the muscimol-impregnated Elvax resulted in characteristic enhancement. Elvax implants without muscimol presented a profile of enhancement characteristic of that in nontreated animals (indicated by dotted line in both panels). C, Inactivation of visual cortex contralateral to the deprived eye, and controls. MD induced an enhancement of OKRs characteristic of that in animals with control Elvax; however, sensitivity dropped to baseline as soon as the deprived eye was opened. Removal of the muscimol-impregnated Elvax enabled a subsequent MD to enhance sensitivity in a characteristic manner. Deprived eye responses (bottom traces) were not affected by $M D$ in either experiment.

cortex. The plasticity shares features with circuits that support learning and memory, because enhanced sensitivity, graded by testing experience and the pattern of MD, can persist after MD. There also appear to be two phases to the plasticity: an induction phase, in which sensitivity is transiently enhanced during MD, and maintenance phase, in which enhanced sensitivity can persist above pre-deprivation baseline after MD. The maintenance phase is also characterized by a permanent "memory" in the form of an immediate enhancement at the time of subsequent $\mathrm{MD}(\mathrm{s})$, as well as a more prolonged enhancement.

Induction seems to proceed in a stepwise manner, the first step of which is a $\sim 10 \%$ increase in spatial frequency sensitivity

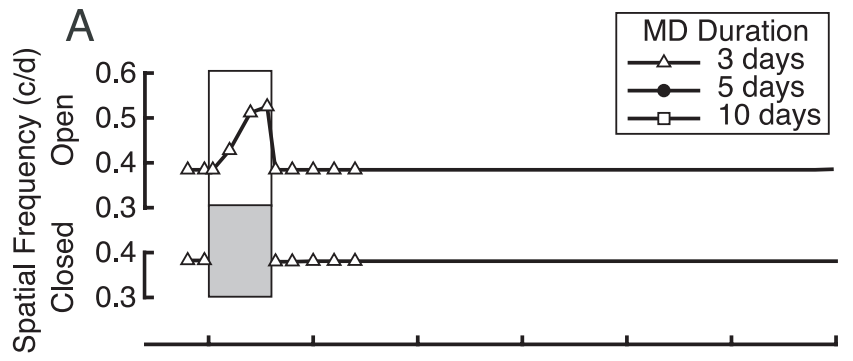

$\mathrm{B}$
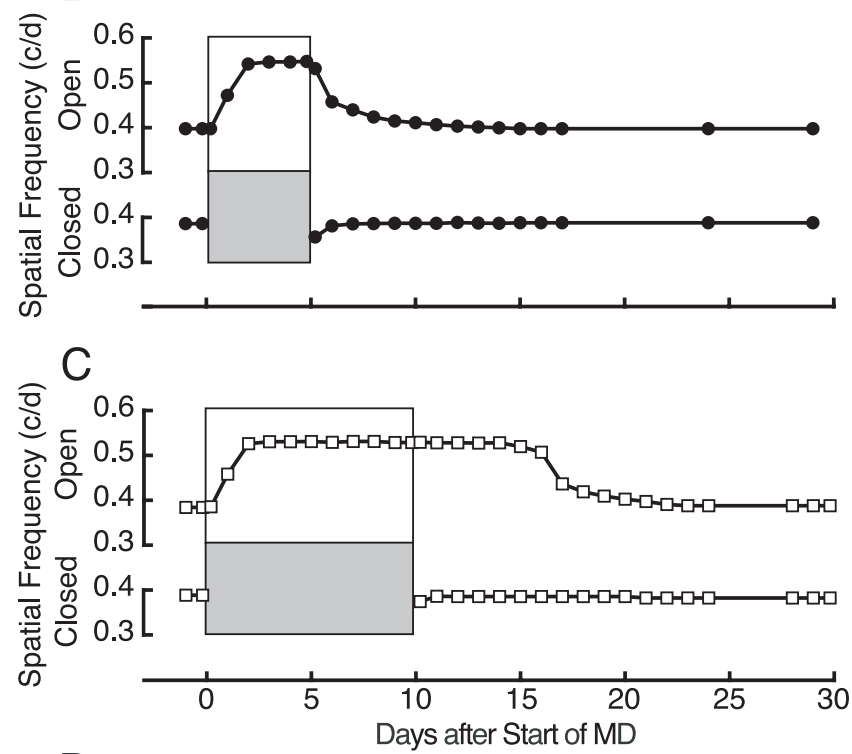

D

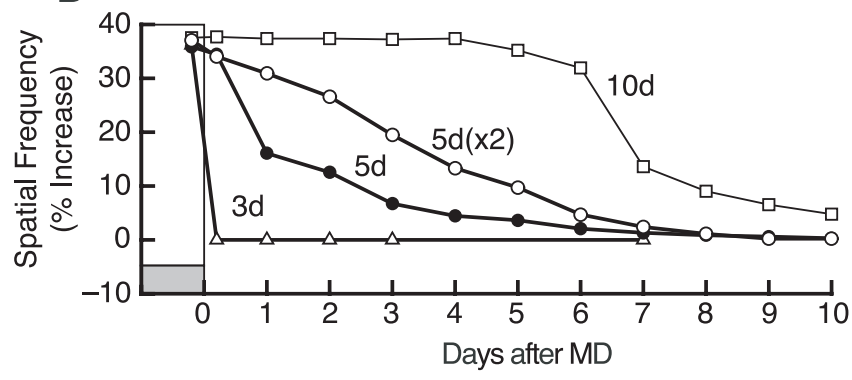

Figure 5. Effect on spatial frequency sensitivity of varying the duration of $\mathrm{MD}$ (indicated by shading). $\boldsymbol{A}$, Three day MD resulted in enhancement through nondeprived eye (top trace) only during the deprivation. $\boldsymbol{B}$, Five day MD resulted in enhanced responses that decremented gradually after MD; replication of experiment in Figure $1 A$. C, Ten day MD both extended the duration of enhanced responses after $M D$, and enabled enhanced sensitivity to persist at maximum. None of the above manipulations affected deprived eye responses ( $\boldsymbol{A}-\boldsymbol{C}$, bottom traces). $\boldsymbol{D}$, Detailed comparison of $3 \mathrm{~d}$ (open triangles), $5 \mathrm{~d}$ (closed circles), twice daily testing for $5 \mathrm{~d}$ (Fig. $3 C$, open circles) and $10 \mathrm{~d}$ (open squares) profiles after MD.

from baseline (Table 1, small enhancement group). A simple asymmetry in interactions between the eyes is sufficient to enable this step, because enucleation results in enhancement to $0.43 \mathrm{c} / \mathrm{d}$ that persists indefinitely, regardless of whether the intact eye is tested or deprived. Because MD combined with bilateral removal of the visual cortex results in the same level of enhancement, only subcortical circuits appear necessary for this step.

The next step, from $\sim 10$ to $\sim 28 \%$ enhancement, is not dependent on daily testing experience (Table 1, Intermediate enhancement, no persistence group), but it does require the cortex, because bilateral lesions, even with daily testing, do not result in an enhancement that surpasses $0.43 \mathrm{c} / \mathrm{d}$. 

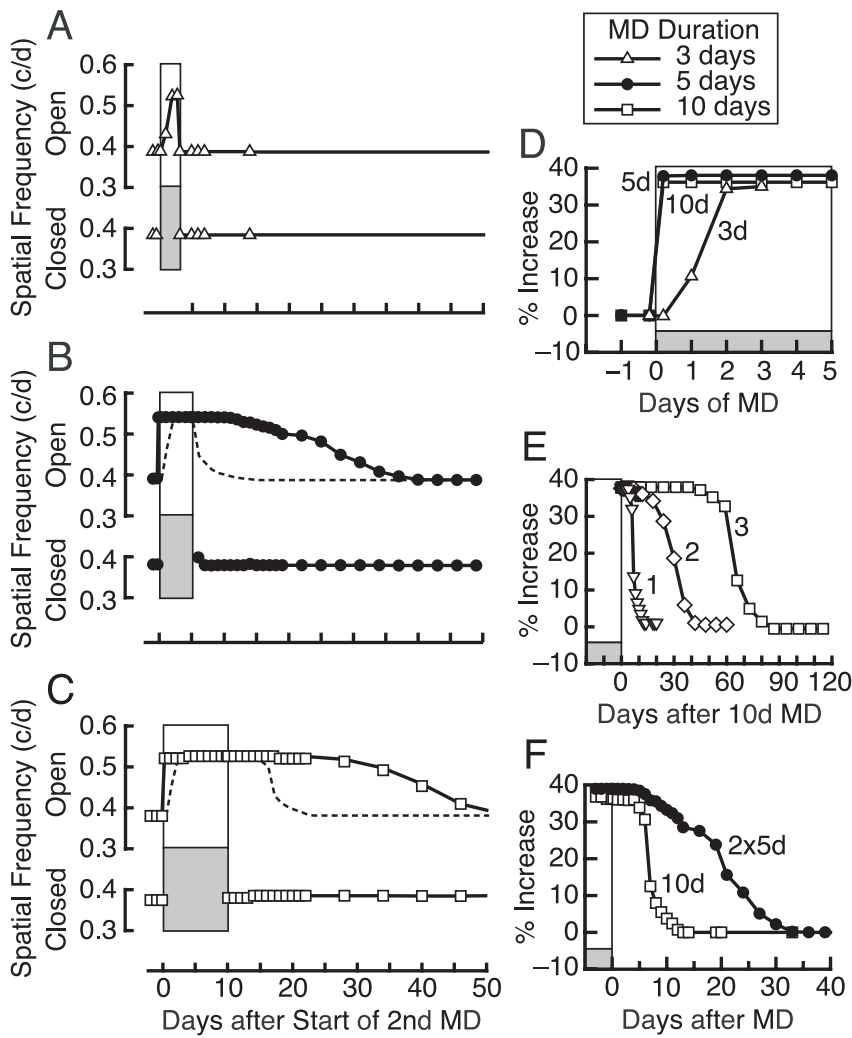

Days after 10d MD

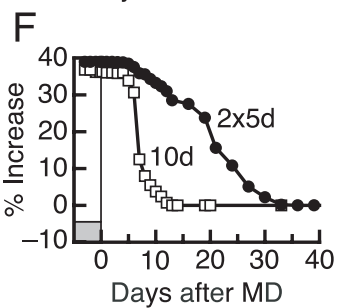

Figure 6. Effect on spatial frequency sensitivity of repeating MD (shading). Traces in $\boldsymbol{A}$-C are a continuation of those in Figure 5, $A-C$ (represented with dotted lines). $\boldsymbol{A}$, Repeating a $3 \mathrm{~d}$ period of MD resulted in a profile of enhancement through nondeprived eyes similar to that after the first MD. $\boldsymbol{B}$, A second $5 \mathrm{~d}$ MD resulted in an immediate maximal enhancement that was maintained at maximal values and remained above baseline far longer than after the first MD. $\boldsymbol{C}$, A second $10 \mathrm{~d} M D$ resulted in a profile similar to $\boldsymbol{B}$, but enhanced responses were maintained even longer. Repeated MD did not affect deprived eye responses ( $\boldsymbol{A}-\boldsymbol{C}$, bottom traces). $\boldsymbol{D}$, Comparison of second 3, 5, and $10 \mathrm{~d}$ MD profiles after MD, in detail. $\boldsymbol{E}$, Comparison of profiles after first (1) (Fig. 5C) second (2) (C), and third (3) periods of $10 \mathrm{~d}$ MD reveals increasing duration of enhancement. $\boldsymbol{F}$, Two, 5 d periods of $\mathrm{MD}(2 \times 5 \mathrm{~d} ; \boldsymbol{B})$ resulted in longer duration enhancement after MD than that after a single $10 \mathrm{~d}$ MD (10d) (Fig. 5C).

The third step, from $\sim 28 \%$ to maximal enhancement at $\sim 37 \%$ (Table 1, Maximal enhancement, with short persistence group), depends on experience-dependent activity in visual cortex, ipsilateral to the deprived eye: active testing during the MD is required to generate maximal enhancement, and cortex is again involved as inactivation of the ipsilateral visual cortex blocks the enhancement. Repeated daily testing at maximal enhancement also appears to be necessary to convert maximal enhancement into a form that persists after MD; $3 \mathrm{~d}$ MD with testing enables sensitivity to reach maximum, but the enhancement does not persist after MD; whereas it does after longer MD. The results of cortical inactivation studies also show that the maintenance of enhanced responses after MD depends on activity in the visual cortex contralateral to the deprived eye. The persistent enhancement is exceptionally dynamic in nature, because increasing testing frequency during the $\mathrm{MD}$, or increasing the duration of $\mathrm{MD}$, progressively extends the duration of enhanced sensitivity (Table 1, Maximal enhancement, with extended persistence group). In addition, repeated MD shows that the maintenance of enhanced sensitivity can be systematically extended, and that the first induction of persistent enhancement leaves a long-term, if not, permanent trace in the form of an immediate maximal enhancement after repeated MD.
Given that the induction of enhancement depends on binocular interactions, we were surprised that enhanced sensitivity after MD was limited to the monocular field and that no change in the directionality of the OKR emerged. These data suggest that if corticofugal pathways to the AOS are involved in the plasticity, only monocular cortical cells are being recruited. This possibility is consistent with the results of Pham et al. (2004), showing that the major physiological and cellular changes occur in the hemisphere ipsilateral to the deprived eye, which is dominated by monocular input.

The effects of adult MD on cortical OD are distinct from those in juvenile animals: Rather than a diminution of responses through the deprived eye, there is an increase in responses through the nondeprived eye. The combination of our previous results, that $\mathrm{MD}$ in adult mice does not affect visual acuity (Prusky and Douglas, 2003), and the results of the present study make a case for the selective association of adult OD plasticity with an enhancement of the OKR. One possibility is that rather than simply improving the sensitivity of a stabilizing visual reflex, a different function, such as visual pursuit, is being enabled by the MD. In the fly, for example, the object tracking system is plastic, whereas the image stabilization system is not (Geiger and Poggio, 1975). Although the analogy of a fixed subcortical pathway for image stabilization, and a plastic cortical pathway seems plausible, the identification of pursuit in the mouse under our current testing conditions is not possible. However, we observed good tracking with grating patches as small as $50^{\circ}$ in width, and although this is much smaller than the full-field stimulation generated by self-motion, it is still large by object-tracking standards. Short-latency ocular tracking of shifts of large visual fields is reported to depend on extrastriate visual cortex in primates (Masson et al., 2001), and therefore, it is also possible that similar extrastriate structures in the mouse are involved in the plasticity described here.

The plasticity we have identified here may also be related to visual perceptual learning in the cortex (Thiele, 2004), a form of adult plasticity in which responses to visual stimulation are gradually enhanced. Indeed, the plasticity enabled by adult MD displays distinct learning-like features, such as improved performance over testing sessions, a slow decay after MD (forgetting), rapid expression of enhancement during a second MD (savings), and longer decay rates after two $5 \mathrm{~d}$ MDs than after one $10 \mathrm{~d}$ MD (distributed Vs massed training). In fact, a form of adult perceptual learning has been linked with binocular visual motion systems ( $\mathrm{Lu}$ et al., 2005).

Physiological studies using visual evoked potentials (VEPs) (Sawtell et al., 2003; Pham et al., 2004) and optical imaging (Hofer et al., 2006) techniques in adult mice have reported increased responses in binocular V1, and our data are in general agreement with these findings. We also found enhancement of behavioral responses through the nondeprived eye, and it is possible that synaptic changes in V1 are the causal basis for the improvement of OKR sensitivity. This interpretation is further supported by the similarities between our results and reports that enhanced signals after MD depend on the duration of deprivation (Sawtell et al., 2003; Pham et al., 2004; Hofer et al., 2006) and on previous experience (He et al., 2006; Hofer et al., 2006). At present, however, there is no direct support for this linkage, and important differences exist between the behavioral and physiological studies.

First, our animals were unrestrained and moved freely during testing, whereas the physiological experiments were completed in restrained, and sometimes anesthetized, animals. These are not 
trivial differences, because our animals were required to produce a functional response to achieve full enhancement, and Pham et al. (2004) have reported that adult OD plasticity is disrupted by some anesthetic regimens. Second, we found that active testing over a $5 \mathrm{~d} \mathrm{MD}$ was necessary to generate an enduring behavioral enhancement, and little or no daily testing was done in the physiological experiments. Consequently, the results of the physiological experiments may be more pertinent to our short-term (induction) plasticity than to our enduring enhancement after MD (maintenance). Third, our measurements involved locating spatial frequency and contrast sensitivity thresholds of a moving grating, whereas other reports of adult OD plasticity used low spatial frequency counterphasing gratings, and differences in the test stimulus could affect the dynamics and location of physiological changes. Fourth, the physiological studies were aimed primarily at the binocular zone of the visual cortex, and although we found that binocular interactions are important, our behavioral effect is manifest only in the monocular segment. The physiological changes, therefore, may be more related to an interaction component than to the usage of visual information in the behavior. In addition, although our data are consistent with the hypothesis that binocular V1 is the locus of the experiencedependent change necessary to enable the maintenance of enhanced responses, this plasticity may itself be dependent on subcortical interocular interactions, and clearly, both visual cortices play a role in the maintenance of enhanced responses. Fifth, the quantitative relationship between the physiology and behavior may be complicated. For example, there is a suggestion of a saturation in the behavior with sensitivity plateauing at $\sim 0.53$ $\mathrm{c} / \mathrm{d}$. If the imaging or VEP studies do not share the same nonlinearity, physiological changes may occur in situations in which there is no behavioral change. Conversely, behavioral assays are often more sensitive than physiological measures. In addition, the state of cortical ocular dominance is not, in and of itself, a predictor of visual function (Murphy and Mitchell, 1987). Finally, past experiments should remind us that physiological changes can be widespread in learning phenomena and that a physiological change in one locus need not have a single behavioral effect

Because both physiological and behavioral signatures of adult OD plasticity have now been identified in a mouse, an animal with distinct advantages as an experimental model, it provides a unique opportunity to identify the structural and functional substrates of an adult form of neural plasticity.

\section{References}

Distler C, Hoffmann KP (1992) Early development of the subcortical and cortical pathway involved in optokinetic nystagmus: the cat as a model for man? Behav Brain Res 49:69-75.

Douglas RM, Alam NM, Silver BD, McGill TJ, Tschetter W, Prusky GT (2005) Independent visual threshold measurements in the two eyes of freely moving rats and mice using a virtual-reality optokinetic system. Vis Neurosci 22:677-684.

Franklin K, Paxinos G (1997) The mouse brain in stereotaxic coordinates. San Diego, CA: Academic.

Geiger G, Poggio T (1975) The orientation of flies towards visual patterns: on the search for the underlying interactions. Biol Cybern 19:39-54.

Giffin F, Mitchell DE (1978) The rate of recovery of vision after early monocular deprivation in kittens. J Physiol (Lond) 274:511-537.

Gordon JA, Stryker MP (1996) Experience-dependent plasticity of binocu- lar responses in the primary visual cortex of the mouse. J Neurosci 16:3274-3286.

Grasse KL, Cynader MS (1988) The effect of visual cortex lesions on vertical optokinetic nystagmus in the cat. Brain Res 455:385-389.

Grasse KL, Cynader MS, Douglas RM (1984) Alterations in response properties in the lateral and dorsal terminal nuclei of the cat accessory optic system following visual cortex lesions. Exp Brain Res 55:69-80.

He HY, Hodos W, Quinlan EM (2006) Visual deprivation reactivates rapid ocular dominance plasticity in adult visual cortex. J Neurosci 26:2951-2955.

Hofer SB, Mrsic-Flogel TD, Bonhoeffer T, Hubener M (2006) Prior experience enhances plasticity in adult visual cortex. Nat Neurosci 9:127-132.

Hoffmann KP, Bremmer F, Thiele A, Distler C (2002) Directional asymmetry of neurons in cortical areas MT and MST projecting to the NOT-DTN in macaques. J Neurophysiol 87:2113-2123.

Hubel DH, Wiesel TN (1970) The period of susceptibility to the physiological effects of unilateral eye closure in kittens. J Physiol (Lond) 206:419-436.

Lu ZL, Chu W, Dosher BA, Lee S (2005) Independent perceptual learning in monocular and binocular motion systems. Proc Natl Acad Sci USA 102:5624-5629.

Masson GS, Busettini C, Yang DS, Miles FA (2001) Short-latency ocular following in humans: sensitivity to binocular disparity. Vision Res 41:3371-3387.

Murphy KM, Mitchell DE (1987) Reduced visual acuity in both eyes of monocularly deprived kittens following a short or long period of reverse occlusion. J Neurosci 7:1526-1536.

Natal CL, Britto LR (1988) The rat accessory optic system: effects of cortical lesions on the directional selectivity of units within the medial terminal nucleus. Neurosci Lett 91:154-159.

Pham TA, Graham SJ, Suzuki S, Barco A, Kandel ER, Gordon B, Lickey ME (2004) A semi-persistent adult ocular dominance plasticity in visual cortex is stabilized by activated CREB. Learn Mem 11:738-747.

Prusky GT, Douglas RM (2003) Developmental plasticity of mouse visual acuity. Eur J Neurosci 17:167-173.

Prusky GT, Ramoa AS (1999) Novel method of chronically blocking retinal activity. J Neurosci Methods 87:105-110.

Prusky GT, West PW, Douglas RM (2000) Behavioral assessment of visual acuity in mice and rats. Vision Res 40:2201-2209.

Prusky GT, Alam NM, Beekman S, Douglas RM (2004) Rapid quantification of adult and developing mouse spatial vision using a virtual optomotor system. Invest Ophthalmol Vis Sci 45:4611-4616.

Sawtell NB, Frenkel MY, Philpot BD, Nakazawa K, Tonegawa S, Bear MF (2003) NMDA receptor-dependent ocular dominance plasticity in adult visual cortex. Neuron 38:977-985.

Schmidt M, Zhang HY, Hoffmann KP (1993) OKN-related neurons in the rat nucleus of the optic tract and dorsal terminal nucleus of the accessory optic system receive a direct cortical input. J Comp Neurol 330:147-157.

Shintani T, Hoshino K, Meguro R, Kaiya T, Norita M (1999) A light and electron microscopic analysis of the convergent retinal and visual cortical projections to the nucleus of the optic tract (NOT) in the pigmented rat. Neurobiology 7:445-460.

Simpson JI (1984) The accessory optic system. Annu Rev Neurosci 7:13-41.

Smith AL, Cordery PM, Thompson ID (1995) Manufacture and release characteristics of Elvax polymers containing glutamate receptor antagonists. J Neurosci Methods 60:211-217.

Smith AL, Parsons CH, Lanyon RG, Bizley JK, Akerman CJ, Baker GE, Dempster AC, Thompson ID, King AJ (2004) An investigation of the role of auditory cortex in sound localization using muscimol-releasing Elvax. Eur J Neurosci 19:3059-3072.

Thiele A (2004) Perceptual learning: is V1 up to the task? Curr Biol 14:R671-R673.

Wagor E, Mangini NJ, Pearlman AL (1980) Retinotopic organization of striate and extrastriate visual cortex in the mouse. J Comp Neurol 193:187-202.

Wiesel TN, Hubel DH (1963) Single-cell responses in striate cortex of kittens deprived of vision in one eye. J Neurophysiol 26:1003-1017. 